\title{
Improved Estimators of Co-efficient of Variation from Bivariate Normal distribution: A Monte Carlo comparison
}

\author{
Archana V. \\ Mangalore University, Karnataka, India \\ archana_stkl@yahoo.com \\ Aruna Rao K. \\ Department of Statistics \\ Mangalore University, Mangalagangothri \\ arunaraomu@gmail.com
}

\begin{abstract}
Co-efficient of variation is a unitless measure of dispersion and is very frequently used in scientific investigations. This has motivated several researchers to propose estimators and tests concerning the coefficient of variation of normal distribution(s). While proposing a class of estimators for the co-efficient of variation of a finite population, Tripathi et al., (2002) suggested that the estimator of co-efficient of variation of a finite population can also be used as an estimator of C.V for any distribution when the sampling design is SRSWR. This has motivated us to propose 28 estimators of finite population coefficient of variation as estimators of co-efficient of variation of one component of a bivariate normal distribution when prior information is available regarding the second component. Cramer Rao type lower bound is derived to the mean square error of these estimators. Extensive simulation is carried out to compare these estimators. The results indicate that out of these 28 estimators, eight estimators have larger relative efficiency compared to the sample co-efficient of variation. The asymptotic mean square errors of the best estimators are derived to the order of $O\left(\frac{1}{n}\right)$ for the benefit of users of co-efficient of variation.
\end{abstract}

Keywords: Co-efficient of variation, Finite population, SRSWR, Bivariate normal distribution, Cramer Rao lower bound.

\section{Introduction}

Co-efficient of Variation (C.V) is a widely used measure of dispersion. It is a relative measure and is unitless. Thus C.V is popular among the researchers and scientists in various disciplines and is defined as, $\frac{\sigma}{\mu}$ where ' $\sigma$ ' refers to the standard deviation and ' $\mu$ ' refers to mean respectively. C.V is used in the analysis of rainfall data (Ananthakrishnan and Soman (1989)) and is equally important as that of the mean rainfall. For comparing the variability in rainfall, C.V is a better measure of dispersion rather than the standard deviation, as the mean rainfall changes across the places and it is quite natural to expect a higher variability when the average rainfall is low. C.V is also used in the analysis of stock market prices. In the stock market data, ' $\mu$ ' refers to mean return and ' $\sigma$ ' refers to volatility in the stock prices. Thus C.V can be interpreted as the volatility per mean return (Verril and Johnson (2007) and Curto and Pinto (2009)). For some of the applications of C.V in the financial analysis see Brief and Owen (1969) and 
Weinraub and Kuhlman (1994). In finance, the C.V can be used as a measure of relative risks (Miller and Karson (1977)) and a test of the equality of the C.V's for two stocks, can help to determine if the two stocks possess the same risk or not. For other applications of C.V refer Nairy and Rao (2003), Bhat and Rao (2007) and some of the references cited therein.

The earliest reported work on C.V dates back to McKay, Pearson and Fieller (1932); wherein they proposed confidence interval for the C.V of the normal distribution. These confidence intervals were improved upon by Hendrick and Robey (1936) and Koopmans et al., (1964). Since then the later works on C.V were concentrated in two directions.

i) Improved Estimators of C.V for the normal distribution.

Some of the works in this area are the following:

Ahmed (1995) proposed shrinkage preliminary test estimator of the co-efficient of variation of a normal distribution when additional sample is available to increase the precision of the estimator. An almost unbiased estimator of the co-efficient of variation was proposed by Breunig (2001) where a bias corrected estimator is provided for the C.V. Liu, Pang and Huang (2006) suggested exact confidence bounds for C.V of a normally distributed population. Three methods for constructing confidence intervals for the co-efficient of variation from normal populations are compared through simulation study by Ng (2006). Mahmoundvand and Hassani (2009) proposed an approximately unbiased estimator for the population $\mathrm{C} . \mathrm{V}$ in a normal distribution. Using this estimator and its variance, two approximate confidence intervals for C.V are also proposed. Other work in this area was by Panichkitkosolkul (2009) who proposed improved confidence intervals for the C.V of a normal distribution.

ii) Tests for equality of C.V's of normal distributions.

Rao and Bhatt (1989) proposed tests for C.V in one and two populations and derived the Edgeworth expansion for the distribution function of sample C.V. The asymptotic robustness of these tests was discussed in Rao and Vidya (1992). On the other hand Singh (1993) uses inverse sample co-efficient of variation (ISCV) and derives the expression for the standard error of these estimators (first order) for various distributions. The paper by Sharma and Krishnan (1994) also uses ISCV to make inference about population C.V. Inverse C.V (ICV) is also the focal point of the paper by Chaturvedi and Rani (1996) where, a sequential procedure is developed inorder to construct a confidence interval of fixed width and pre-assigned coverage probability for the inverse of the C.V of a normal distribution. In addition to the above tests of C.V for one and two normal populations, tests are also available for the case of ' $k$ ' normal populations. Bennett (1976) proposed a likelihood ratio test for equality of $\mathrm{C}$.V of ' $\mathrm{k}$ ' independent normal populations using the transformed sample C.V's which was later modified by Shafer and Sullivan (1986). Doornbos and Dijkstra (1983) proposed two tests: a likelihood ratio test and a non-central ' $t$ ' test for this problem. Gupta and Ma (1996) proposed score test for the equality of C.V's of 'k' normal populations. Singh (1993) observed that the Taylor series expansion 
of ISCV involves less number of terms than the sample C.V. This has motivated Nairy and Rao (2003) to propose three new tests for the equality of ICV of ' $\mathrm{k}$ ' independent normal populations. Verril and Johnson (2007) proposed confidence bounds and hypothesis tests for C.V of ' $\mathrm{k}$ ' independent normal distribution. The likelihood ratio test proposed in this paper differs from the test given in Gupta and Ma (1996) and Nairy and Rao (2003) and provides a rigorous proof of the $\chi^{2}$-approximation to the asymptotic null distribution of the test statistic. Forkman (2009) proposed an explicit estimator and tests for common co-efficients of variation of ' $\mathrm{k}$ ' independent normal distributions which is an extension of the work of Ahmed (2002). All the previous references relate to the case of samples from independent normal distributions. This is extended for the non-iid case by Curto and Pinto (2009), where they develop tests for equality of C.V's for a time series data.

Although the above works relate to $\mathrm{C} . \mathrm{V}$ of one or more normal distributions, the estimation of C.V did not draw the attention of the researchers in finite population for a long time. It was Das and Tripathi (1981 a, b), who first proposed the estimator of population $\mathrm{C} . \mathrm{V}$ when samples were selected using simple random sampling without replacement (SRSWOR). Subsequently, Das and Tripathi $(1992,93)$ proposed a class of estimators for population C.V using the idea of ratio and regression estimators. Tripathi et al., (2002) extended this class by defining a class of estimators satisfying certain conditions. In a certain sense this is an abstract class where, in the proposition of this class no reference is made to any particular estimator. They obtained the optimum estimators belonging to this class and the estimators of Das and Tripathi (1981 a, b) are members of this class. Patel and Shah (2009) conducted a Monte Carlo comparison of the several estimators of finite population C.V under SRSWOR. The problem of estimation of C.V has also been discussed by Rajyaguru and Gupta $(2002,2006)$ where in they propose new estimators of C.V under simple random sampling as well as stratified random sampling.

Tripathi et al., (2002) noted that the estimators of C.V proposed under SRS with replacement (SRSWR) scheme can also be considered as estimators of C.V for any distributions. This idea is also used in Maiti (2009) and Maiti and Tripathi (2009) to propose new shrinkage-type estimators of population variance of some univariate and bivariate populations. This has motivated us to propose improved estimators of $\mathrm{C} . \mathrm{V}$ of a bivariate normal distribution when auxiliary information is available regarding the second component of the bivariate normal distribution. In this paper we propose 28 estimators of finite population C.V under SRSWR as estimators of C.V of one component of a bivariate normal distribution when prior information is available regarding the second component (in terms of mean, variance or C.V). Archana and Rao (2011) observed that we can always propose an improved estimator of any parameter ' $\theta$ ' using the regression estimators. A question which remains unanswered is that, what would be the lower bound for the variance of such type of estimators? A partial answer to this question is provided in this paper by deriving the Cramer Rao (C.R) type lower bound for the variance of any 
estimator of C.V from a bivariate normal distribution. We have derived C.R lower bound for the variance of the estimators of C.V, of a bivariate normal distribution. Extensive simulation is carried out to compare these estimators. The finite sample performance of the estimators indicate that out of these 28 estimators, eight estimators perform well (having smaller mean square error) compared to the sample C.V.

The organization of the paper is as follows:

In section 2, we describe 28 estimators of C.V proposed under SRSWR as estimators of C.V of a bivariate normal distribution. In section 3, we drive the C.R type lower bound for the variance of the estimators of C.V. In section 4, we compare finite sample performance of these estimators using extensive simulation. The concluding remarks are provided in section 5 .

\section{New estimators of C.V of bivariate normal distribution}

Let the paired observations $\left(\mathrm{x}_{1}, \mathrm{y}_{1}\right), \ldots,\left(\mathrm{x}_{\mathrm{n}}, \mathrm{y}_{\mathrm{n}}\right)$ be a sample from a bivariate population. When the design is SRSWR, this can also be viewed as a sample from any bivariate distribution (Tripathi et al., (2002)). Thus treat this as a sample of size ' $n$ ' from a bivariate normal distribution with parameters $\left(\mu_{x}, \sigma_{x}^{2}, \mu_{y}, \sigma_{y}^{2}, \rho\right)$. Here $\mu$ refers to mean and $\sigma^{2}$ refers to variance of the random variable under consideration and $\rho$ refers to the correlation co-efficient. To make the things explicit, let $\mathrm{Y}$ denote the response variable and $\mathrm{X}$, the auxiliary variable. The parameter of interest is $\theta_{y}=\frac{\sigma_{y}}{\mu_{y}}$, the C.V of the response variable ' $\mathrm{Y}$ '. For a finite population, various estimators of $\theta_{y}$ are proposed in the past (see Archana and Rao (2011), Tripathi et al. (2002)). In this paper we propose these estimators as estimators of $\theta_{y}$ from bivariate normal distributions. The estimators are suitably modified for the bivariate normal distribution to accommodate the fact that all odd ordered moments are zero and co-efficient of kurtosis is equal to 3.

\subsection{Sample C.V $\left(\hat{\theta}_{y_{1}}\right)$}

The sample C.V is obtained by using the sample mean and sample standard deviation as an estimators of the denominator and numerator respectively. It is given by

$$
\hat{\theta}_{y_{1}}=\frac{s_{y}}{\bar{y}}
$$

where $\bar{y}$ is the sample mean and $s_{y}{ }^{2}$ is the sample variance and are given by,

$$
\bar{y}=\frac{\sum y_{i}}{n} \text { and } s_{y}^{2}=\frac{\sum\left(y_{i}-\bar{y}\right)^{2}}{n-1} .
$$

Note: This sample C.V also turns out to be maximum likelihood estimate (MLE) of $\theta_{y}$ for a bivariate normal distribution. 
In the sequel, we propose the estimators of C.V. under SRSWR as estimators of C.V of a bivariate normal distribution.

2.2. Archana and Rao (2011) proposed a class of ratio and product estimators of population C.V and a class of regression/regression type estimators of population C.V under simple random sampling. Here we list below, some of the best estimators considered by them.

2.2.1. Regression type estimator $\left(\hat{\theta}_{y_{2}}\right)$ is given by,

$$
\hat{\theta}_{y_{2}}=\frac{\left(s_{y}^{2}+b_{2}\left(\sigma_{x}^{2}-s_{x}^{2}\right)\right)^{\frac{1}{2}}}{\left(\bar{y}+b_{1}(\bar{X}-\bar{x})\right)}
$$

where $B_{1}=\frac{\sigma_{x y}}{\sigma_{x x}}$ and $B_{2}=\left\{\frac{\sigma_{x x y y}-\sigma_{x x} \sigma_{y y}}{\sigma_{x x x x}-\left(\sigma_{x x}\right)^{2}}\right\}$ under simple random sampling with replacement.

Under normal distribution the expression for $\mathrm{B}_{1}$ and $\mathrm{B}_{2}$ simplifies to,

$$
B_{1}=\frac{\sigma_{x y}}{\sigma_{x x}} \text { and } B_{2}=\frac{\left(\sigma_{x y}\right)^{2}}{\left(\sigma_{x x}\right)^{2}}
$$

\subsubsection{Regression estimators $\left(\hat{\theta}_{y_{3}}\right)$}

$$
\hat{\theta}_{y_{3}}=\frac{s_{y}}{\bar{y}}+b_{3}\left(\frac{\sigma_{x}}{\bar{X}}-\frac{s_{x}}{\bar{x}}\right)
$$

where $B_{3}=\frac{\left\{\frac{\left(\sigma_{x x y y}-\sigma_{x x} \sigma_{y y}\right)}{4 \bar{X} \bar{Y} \sigma_{x} \sigma_{y}}-\frac{\sigma_{x y y} \sigma_{x}}{2 \sigma_{y} \bar{Y} \bar{X}^{2}}-\frac{\sigma_{x x y} \sigma_{y}}{2 \sigma_{x} \bar{X} \bar{Y}^{2}}+\frac{\sigma_{x y} \sigma_{x} \sigma_{y}}{\bar{X}^{2} \bar{Y}^{2}}\right\}}{\left\{\frac{\left(\sigma_{x x x x}-\left(\sigma_{x x}\right)^{2}\right)}{4 \bar{X}^{2} \sigma_{x x}}+\left(\frac{\sigma_{x}}{\bar{X}}\right)^{4}-\frac{\sigma_{x x x}}{\bar{X}^{3}}\right\}}$ under SRSWR.

Under normal distribution this simplifies to, $B_{3}=\frac{\left\{\frac{\left(\sigma_{x y}{ }^{2}\right)}{2 \bar{X} \bar{Y} \sigma_{x} \sigma_{y}}+\frac{\sigma_{x y} \sigma_{x} \sigma_{y}}{\bar{X}^{2} \bar{Y}^{2}}\right\}}{\left\{\frac{\sigma_{x x}}{2 \bar{X}^{2}}+\left(\frac{\sigma_{x}}{\bar{X}}\right)^{4}\right\}}$

\subsubsection{Ratio estimator $\left(\hat{\theta}_{y_{4}}\right)$}

$$
\hat{\theta}_{y_{4}}=\frac{s_{y}}{\bar{y}} \times \frac{\bar{X}}{\bar{x}}
$$

This ratio estimator uses information on mean of the auxiliary variable. 
2.2.4. Ratio estimator $\left(\hat{\theta}_{y_{5}}\right)$

$$
\hat{\theta}_{y_{4}}=\frac{s_{y}}{\bar{y}} \times\left(\frac{S_{x} / \bar{X}}{s_{x} / \bar{x}}\right)
$$

This ratio estimator uses information on C.V of the auxiliary variable. (For details see Archana and Rao (2011))

2.3. Das and Tripathi (1980) proposed a class of hybrid estimators (mixture of ratio and regression estimators) of C.V under simple random sampling. This class of estimators is generalized by Tripathi et al., (2002). Some of the best estimators considered by them (under simple random sampling) are proposed as estimators of C.V for bivariate normal distribution and are listed below. The finer details are available in their paper.

\subsection{1.}

$\hat{\theta}_{y_{6}}=\hat{C}_{y} \times\left(\frac{\bar{x}}{\bar{X}}\right) \times\left(\frac{\hat{\sigma}_{x}^{2}}{\sigma_{x}^{2}}\right)^{\alpha} \quad$ where $\alpha=-0.3542$ and $\hat{\mathrm{C}}_{\mathrm{y}}=\frac{s_{y}}{\bar{y}}$

\subsection{2.}

$\hat{\theta}_{y_{7}}=\hat{C}_{y} \times\left(\frac{\bar{x}}{\bar{X}}\right)^{\alpha_{1}} \times\left(\frac{\hat{\sigma}_{x}}{\sigma_{x}}\right) \quad$ where $\alpha_{1}=-1.32105$

\subsection{2.}

$\hat{\theta}_{y_{8}}=\hat{C}_{y} \times\left(\frac{\bar{x}}{\bar{X}}\right) \times\left(\frac{\sigma_{x}}{\hat{\sigma}_{x}}\right)$

\subsection{3.}

$\hat{\theta}_{y_{9}}=\frac{\hat{\sigma}_{y}-\alpha_{2}\left(\hat{\sigma}_{x}-\sigma_{x}\right)}{\bar{y}} \quad$ where $\alpha_{2}=1.32105$

\subsection{4.}

$\hat{\theta}_{y_{10}}=\hat{C}_{y}-\left(\frac{\hat{\mathrm{C}}_{\mathrm{x}}}{\mathrm{C}_{\mathrm{x}}}\right) \quad$ where $\hat{\mathrm{C}}_{\mathrm{x}}=\frac{\mathrm{s}_{\mathrm{x}}}{\overline{\mathrm{x}}}$ 


\subsection{5.}

$$
\hat{\theta}_{y_{11}}=\hat{C}_{y}-\hat{\partial}_{1}(\overline{\mathrm{x}}-\overline{\mathrm{X}})-\hat{\partial}_{2}\left(\mathrm{~s}_{\mathrm{x}}^{2}-\sigma_{\mathrm{x}}^{2}\right)
$$

where $\hat{\partial}_{1}=\frac{\bar{X} \hat{C}_{y}}{\hat{\Delta} \sigma_{x}^{2}}\left[\frac{m_{3}(x)}{\sigma_{x}^{3}} \hat{A}_{2}^{*}-\hat{A}_{1}^{*} \frac{\sigma_{x}}{\bar{X}}\right]$ and

$$
\hat{\partial}_{2}=\frac{\bar{X} \hat{C}_{y}}{\hat{\Delta} \sigma_{x}^{3}}\left[\frac{m_{3}(x)}{\bar{X} \sigma_{x}^{2}} \hat{A}_{1}^{*}-\hat{A}_{2}^{*}\left\{\frac{m_{4}(x)}{\sigma_{x}^{4}}-1\right\}\right]
$$

Under normal distribution this expression simplifies to,

$$
\hat{\partial}_{1}=\frac{-\sigma_{x y}{ }^{2} \hat{C}_{y}}{2 \sigma_{x}^{3} \sigma_{y y}} \quad \text { and } \quad \hat{\partial}_{2}=\frac{\hat{C}_{y} \sigma_{x y}}{\sigma_{x}^{3} \bar{Y}} .
$$

(See Tripathi et al., (2002) for further details.)

2.3.6.

$\hat{\theta}_{y_{12}}=\frac{\hat{\sigma}_{y}-\left(\hat{\sigma}_{x}-\sigma_{x}\right)}{\overline{\mathrm{y}}}$

2.3.7.

$$
\hat{\theta}_{y_{13}}=\hat{C}_{y} \times\left[1+\hat{\lambda}_{1}^{*}\left(\frac{\overline{\mathrm{x}}}{\overline{\mathrm{X}}}-1\right)+\hat{\lambda}_{2}^{*}\left(\frac{\mathrm{s}_{\mathrm{x}}^{2}}{\sigma_{\mathrm{x}}^{2}}-1\right)\right]
$$

where $\hat{\lambda}_{1}^{*}=\frac{\bar{X}^{2}}{\sigma_{x}^{2}} \frac{\left[\frac{m_{3}(x)}{\sigma_{x}^{3}} \hat{A}_{1}^{*}-\hat{A}_{2}^{*}\left\{\frac{m_{4}(x)}{\sigma_{x}^{4}}-1\right\}\right]}{\hat{\Delta}}$

and $\quad \hat{\lambda}_{2}^{*}=\frac{\bar{X}}{\sigma_{x}} \frac{\left[\frac{m_{3}(x)}{\bar{X} \sigma_{x}^{2}} \hat{A}_{2}^{*}-\hat{A}_{1}^{*} \frac{\sigma_{x}}{\bar{X}}\right]}{\hat{\Delta}}$ respectively.

Under normal distribution this simplifies to,

$$
\hat{\lambda}_{1}^{*}=\frac{\sigma_{x y} \bar{X}}{\sigma_{x x} \bar{Y}} \quad \text { and } \quad \hat{\lambda}_{2}^{*}=\frac{-\sigma_{x y}{ }^{2}}{2 \sigma_{x x} \sigma_{y y}} .
$$

\subsection{8.}

$\hat{\theta}_{y_{14}}=\hat{C}_{y}+\hat{\lambda}_{1}(\overline{\mathrm{x}}-\overline{\mathrm{X}})+\hat{\lambda}_{2}\left(\mathrm{~s}_{\mathrm{x}}^{2}-\sigma_{\mathrm{x}}^{2}\right)$

where $\hat{\lambda}_{1}=\hat{\lambda}_{1}^{*} \frac{\hat{C}_{y}}{\bar{X}}$ and $\hat{\lambda}_{2}=\hat{\lambda}_{2}^{*} \frac{\hat{C}_{y}}{\sigma_{x}^{2}}$.

Under normal distribution $\hat{\lambda}_{1}=\frac{\sigma_{x y} \hat{C}_{y}}{\sigma_{x x} \bar{Y}}$ and $\hat{\lambda}_{2}=\frac{-\sigma_{x y}{ }^{2} \hat{C}_{y}}{2\left(\sigma_{x x}\right)^{2} \sigma_{y y}}$. 


\subsection{9.}

$\hat{\theta}_{y_{15}}=\hat{C}_{y} \times\left(\frac{\bar{x}}{\bar{X}}\right)^{\hat{\lambda}_{1}^{*}}$

2.3.10. $\hat{\theta}_{y_{16}}=\frac{\left(s_{y}{ }^{2}+b_{5}\left(\sigma_{x}{ }^{2}-s_{x}{ }^{2}\right)\right)^{\frac{1}{2}}}{\left(\bar{y}+b_{4}(\bar{X}-\bar{x})\right)}$

where $B_{4}=\frac{\sigma_{x y}}{\sigma_{x x}}$ and $B_{5}=\left(\frac{\sigma_{x y}}{\sigma_{x x}}\right)^{2}$.

(See Patel and Shah (2009) for further details.)

2.4. Generally while proposing regression estimators, sample C.V is used to construct a regression estimator. Archana and Rao (2011) noted that any estimator of a parameter (including regression estimator) can be used to construct a new regression estimator. Here we use the estimator $\hat{\theta}_{y_{11}}$ (of Tripathi et al., (2002)) to construct a new regression estimator. These classes of estimators are listed below.

\subsection{1.}

$\hat{\theta}_{y_{17}}=\frac{s_{y}}{\bar{y}}-\hat{\partial}_{1}(\bar{x}-\bar{X})-\hat{\partial}_{2}\left(s_{x x}-\sigma_{x x}\right)+b_{6}(\bar{X}-\bar{x})$

where $B_{6}=\frac{\left(-\frac{\sigma_{x y} \sigma_{y}}{\bar{Y}^{2}}-\hat{\partial}_{1} \sigma_{x x}\right)}{\sigma_{x x}}$ under normal distributi on.

\subsection{2.}

$\hat{\theta}_{y_{18}}=\frac{s_{y}}{\bar{y}}-\hat{\partial}_{1}(\bar{x}-\bar{X})-\hat{\partial}_{2}\left(s_{x x}-\sigma_{x x}\right)+b_{7}\left(\sigma_{x}^{2}-s_{x}^{2}\right)$

where $B_{7}=\frac{\left(\frac{\sigma_{x y}{ }^{2}}{\bar{Y} \sigma_{y}}-2 \hat{\partial}_{2} \sigma_{x x}{ }^{2}\right)}{2 \sigma_{x x}{ }^{2}}$ under normal distribution.

\subsection{3.}

$\hat{\theta}_{y_{19}}=\frac{s_{y}}{\bar{y}}-\hat{\partial}_{1}(\bar{x}-\bar{X})-\hat{\partial}_{2}\left(s_{x x}-\sigma_{x x}\right)+b_{8}\left(\frac{\sigma_{x}}{\bar{X}}-\frac{s_{x}}{\bar{x}}\right)$

where $B_{8}=\frac{\left(\frac{\sigma_{x y}{ }^{2}}{4 \bar{X} \bar{Y} \sigma_{x} \sigma_{y}}+\frac{\sigma_{x y} \sigma_{x} \sigma_{y}}{\bar{X}^{2} \bar{Y}^{2}}+\frac{\hat{\partial}_{1} \sigma_{x x} \sigma_{x}}{\bar{X}^{2}}-2 \hat{\partial}_{2} \sigma_{x x}{ }^{2}\right)}{\left\{\frac{\sigma_{x x}}{2 \bar{X}^{2}}+\left(\frac{\sigma_{x}}{\bar{X}}\right)^{4}\right\}}$ under normal distribution. 


\subsection{4.}

$\hat{\theta}_{y_{20}}=\frac{s_{y}}{\bar{y}}-\hat{\partial}_{1}(\bar{x}-\bar{X})-\hat{\partial}_{2}\left(s_{x x}-\sigma_{x x}\right)+b_{6}(\bar{X}-\bar{x})+b_{9}(\bar{X}-\bar{x})$

where $B_{9}=\frac{\left[\frac{-\sigma_{x y} \sigma_{y}}{\bar{Y}^{2}}-\delta_{1} \sigma_{x x}+B_{6} \sigma_{x x}\right]}{\sigma_{x x}}$ under normal distribution.

\subsection{5.}

$\hat{\theta}_{y_{21}}=\frac{s_{y}}{\bar{y}}-\hat{\partial}_{1}(\bar{x}-\bar{X})-\hat{\partial}_{2}\left(s_{x x}-\sigma_{x x}\right)+b_{6}(\bar{X}-\bar{x})+b_{10}\left(\sigma_{x}^{2}-s_{x}^{2}\right)$

where $B_{10}=\frac{\left[\frac{\sigma_{x y}^{2}}{\bar{Y} \sigma_{y}}-2 \hat{\delta}_{2} \sigma_{x x}^{2}\right]}{2 \sigma_{x x}^{2}}$ under normal distribution.

\subsection{6.}

$\hat{\theta}_{y_{22}}=\frac{s_{y}}{\bar{y}}-\hat{\partial}_{1}(\bar{x}-\bar{X})-\hat{\partial}_{2}\left(s_{x x}-\sigma_{x x}\right)+b_{6}(\bar{X}-\bar{x})+b_{11}\left(\frac{\sigma_{x}}{\bar{X}}-\frac{s_{x}}{\bar{x}}\right)$

where $\quad B_{11}=\frac{\left[\frac{\sigma_{x y}{ }^{2}}{2 \bar{X} \bar{Y} \sigma_{x} \sigma_{y}}+\frac{\sigma_{x y} \sigma_{x} \sigma_{y}}{\bar{X}^{2} \bar{Y}^{2}}-\frac{\sigma_{x x} \sigma_{x}}{\bar{X}^{2}}-\frac{\hat{\delta}_{2} \sigma_{x x}{ }^{2}}{\bar{X} \sigma_{x}}-\frac{B_{6} \sigma_{x x} \sigma_{x}}{\bar{X}^{2}}\right]}{\frac{\sigma_{x x}}{2 \bar{X}^{2}}+\left(\frac{\sigma_{x}}{\bar{X}}\right)^{4}}$

distribution.

\subsection{7.}

$\hat{\theta}_{y_{23}}=\frac{s_{y}}{\bar{y}}-\hat{\partial}_{1}(\bar{x}-\bar{X})-\hat{\partial}_{2}\left(s_{x x}-\sigma_{x x}\right)+b_{7}\left(\sigma_{x}^{2}-s_{x}^{2}\right)+b_{12}(\bar{X}-\bar{x})$

where $B_{12}=\frac{\left[\frac{-\sigma_{x y} \sigma_{y}}{\bar{Y}^{2}}-\hat{\delta}_{1} \sigma_{x x}\right]}{\sigma_{x x}}$ under normal distribution.

\subsection{8.}

$\hat{\theta}_{y_{24}}=\frac{s_{y}}{\bar{y}}-\hat{\partial}_{1}(\bar{x}-\bar{X})-\hat{\partial}_{2}\left(s_{x x}-\sigma_{x x}\right)+b_{7}\left(\sigma_{x}^{2}-s_{x}^{2}\right)+b_{13}\left(\sigma_{x}^{2}-s_{x}^{2}\right)$

where $B_{13}=\frac{\left[\frac{\sigma_{x y}{ }^{2}}{\bar{Y} \sigma_{y}}-2 \hat{\delta}_{2} \sigma_{x x}{ }^{2}+2 B_{7} \sigma_{x x}{ }^{2}\right]}{2 \sigma_{x x}{ }^{2}}$ under normal distribution. 


\subsection{9.}

$\hat{\theta}_{y_{25}}=\frac{s_{y}}{\bar{y}}-\hat{\partial}_{1}(\bar{x}-\bar{X})-\hat{\partial}_{2}\left(s_{x x}-\sigma_{x x}\right)+b_{7}\left(\sigma_{x}^{2}-s_{x}^{2}\right)+b_{14}\left(\frac{\sigma_{x}}{\bar{X}}-\frac{s_{x}}{\bar{x}}\right)$

where $\quad B_{14}=\frac{\left[\frac{\sigma_{x y}{ }^{2}}{2 \bar{X} \bar{Y} \sigma_{x} \sigma_{y}}+\frac{\sigma_{x y} \sigma_{x} \sigma_{y}}{\bar{X}^{2} \bar{Y}^{2}}+\hat{\delta}_{1} \frac{\sigma_{x x} \sigma_{x}}{\bar{X}^{2}}-\frac{\hat{\delta}_{2} \sigma_{x x}{ }^{2}}{\bar{X} \sigma_{x}}+B_{7} \frac{\sigma_{x x}{ }^{2}}{\bar{X} \sigma_{x}}\right]}{\frac{\sigma_{x x}}{2 \bar{X}^{2}}+\left(\frac{\sigma_{x}}{\bar{X}}\right)^{4}}$ under normal distribution.

\subsubsection{0.}

$\hat{\theta}_{y_{26}}=\frac{s_{y}}{\bar{y}}-\hat{\partial}_{1}(\bar{x}-\bar{X})-\hat{\partial}_{2}\left(s_{x x}-\sigma_{x x}\right)+b_{8}\left(\frac{\sigma_{x}}{\bar{X}}-\frac{s_{x}}{\bar{x}}\right)+b_{15}(\bar{X}-\bar{x})$

where $B_{15}=\frac{\left[\frac{-\sigma_{x y} \sigma_{y}}{\bar{Y}^{2}}-\hat{\delta}_{1} \sigma_{x x}-B_{8} \frac{\sigma_{x x} \sigma_{x}}{\bar{X}^{2}}\right]}{\sigma_{x x}}$ under normal distribution.

\subsubsection{1.}

$\hat{\theta}_{y_{27}}=\frac{s_{y}}{\bar{y}}-\hat{\partial}_{1}(\bar{x}-\bar{X})-\hat{\partial}_{2}\left(s_{x x}-\sigma_{x x}\right)+b_{8}\left(\frac{\sigma_{x}}{\bar{X}}-\frac{s_{x}}{\bar{x}}\right)+b_{16}\left(\sigma_{x}^{2}-s_{x}^{2}\right)$

where $B_{16}=\frac{\left[\frac{\sigma_{x y}{ }^{2}}{\bar{Y} \sigma_{y}}-2 \hat{\delta}_{2} \sigma_{x x}{ }^{2}+B_{8} \frac{\sigma_{x x}{ }^{2}}{\bar{X} \sigma_{x}}\right]}{2 \sigma_{x x}{ }^{2}}$ under normal distribution.

\subsubsection{2.}

$\hat{\theta}_{y_{28}}=\frac{s_{y}}{\bar{y}}-\hat{\partial}_{1}(\bar{x}-\bar{X})-\hat{\partial}_{2}\left(s_{x x}-\sigma_{x x}\right)+b_{8}\left(\frac{\sigma_{x}}{\bar{X}}-\frac{s_{x}}{\bar{x}}\right)+b_{17}\left(\frac{\sigma_{x}}{\bar{X}}-\frac{s_{x}}{\bar{x}}\right)$

where

$$
B_{17}=\frac{\left[\frac{\sigma_{x y}^{2}}{2 \bar{X} \bar{Y} \sigma_{x} \sigma_{y}}+\frac{\sigma_{x y} \sigma_{x} \sigma_{y}}{\bar{X}^{2} \bar{Y}^{2}}+\frac{\hat{\delta}_{1} \sigma_{x x} \sigma_{x}}{\bar{X}^{2}}-\frac{\hat{\delta}_{2} \sigma_{x x}{ }^{2}}{\bar{X} \sigma_{x}}+B_{8} \frac{\sigma_{x x}}{2 \bar{X}^{2}}+B_{8}\left(\frac{\sigma_{x}}{\bar{X}}\right)^{4}\right]}{\frac{\sigma_{x x}}{2 \bar{X}^{2}}+\left(\frac{\sigma_{x}}{\bar{X}}\right)^{4}}
$$

under normal distribution.

The expressions for the asymptotic mean square errors of the best 8 estimators of C.V are derived to the order of $O\left(\frac{1}{n}\right)$ under normal distribution by the authors. The mean square errors of these best estimators are presented in Appendix A. 


\section{Lower bound for the variance of the estimator of $\theta_{y}$}

Archana and Rao (2011) observed that if $\hat{\theta}_{y}$ is any estimator of ' $\theta$ ', a regression estimator of the form $\hat{\theta}_{y}+\hat{\beta}\left(\eta_{x}-\hat{\eta}_{x}\right)$ can be obtained which has got a smaller mean square error compared to $\hat{\theta}_{y}$. This estimator can be taken as the estimator of ' $\theta$ ' and one can propose another regression estimator with a smaller mean square error. A question that needs to be addressed is that, can we propose a lower bound for the variance of the estimator of ' $\theta$ '. In this section, we derive the Cramer Rao (C.R) type lower bound for the variance of the estimator of $\theta_{\mathrm{y}}$. Let $g\left(\mu_{x}, \sigma_{x}, \mu_{y}, \sigma_{y}, \rho\right)$ denote the fisher information matrix for the parameters of the bivariate normal distribution (see Kotz, Balakrishnan and Johnson (2000, p294)) and is given by,

$$
\mathrm{I}\left(\mu_{\mathrm{x}}, \sigma_{\mathrm{x}}, \mu_{\mathrm{y}}, \sigma_{\mathrm{y}}, \rho\right)=\left[\begin{array}{ccccc}
\frac{1}{\sigma_{\mathrm{xx}}\left(1-\rho^{2}\right)} & 0 & \frac{-\rho}{\sigma_{\mathrm{x}} \sigma_{\mathrm{y}}\left(1-\rho^{2}\right)} & 0 & 0 \\
0 & \frac{2-\rho^{2}}{\sigma_{\mathrm{xx}}\left(1-\rho^{2}\right)} & 0 & \frac{-\rho^{2}}{\sigma_{\mathrm{x}} \sigma_{\mathrm{y}}\left(1-\rho^{2}\right)} & \frac{-\rho}{\sigma_{\mathrm{x}}\left(1-\rho^{2}\right)} \\
\frac{-\rho}{\sigma_{\mathrm{x}} \sigma_{\mathrm{y}}\left(1-\rho^{2}\right)} & 0 & \frac{1}{\sigma_{\mathrm{yy}}\left(1-\rho^{2}\right)} & 0 & 0 \\
0 & \frac{-\rho^{2}}{\sigma_{\mathrm{x}} \sigma_{\mathrm{y}}\left(1-\rho^{2}\right)} & 0 & \frac{2-\rho^{2}}{\sigma_{\mathrm{yy}}\left(1-\rho^{2}\right)} & \frac{-\rho}{\sigma_{\mathrm{y}}\left(1-\rho^{2}\right)} \\
0 & \frac{-\rho}{\sigma_{\mathrm{x}}\left(1-\rho^{2}\right)} & 0 & \frac{-\rho}{\sigma_{y}\left(1-\rho^{2}\right)} & \frac{1+\rho^{2}}{\left(1-\rho^{2}\right)^{2}}
\end{array}\right]
$$

The C.R. type lower bound for an unbiased estimator of $\mathrm{g}($.$) is given by(see Lehmann and$ Casella (1998)),

$$
=\left(\frac{\partial g(\theta)}{\partial \theta}\right)^{\prime}(n I(\theta))^{-1}\left(\frac{\partial g(\theta)}{\partial \theta}\right)
$$

where $\theta=\left(\mu_{x}, \sigma_{x}, \mu_{y}, \sigma_{y}, \rho\right)$

After some simplification, C.R lower bound is given by,

$$
=\frac{\theta_{y}{ }^{4}}{n}\left(1-2 \rho^{2}\right)+\frac{1}{2 n} \theta_{y}{ }^{2}
$$

In general, unbiased estimator of $\theta_{\mathrm{y}}$ does not exists and this lower bound can be treated as the lower bound for the asymptotic mean square error of an estimator of $\hat{\theta}_{y}$ which is unbiased to the order of $O\left(\frac{1}{n}\right)$. 


\section{Finite sample comparison of the estimators of C.V.}

\subsection{Simulation experiment}

A simulation experiment is carried out to compare the MSE's of various estimators. The estimated MSE's using simulation are more accurate and thus gives a clear comparison of the efficiency of the various estimators. For this purpose a sample of size ' $n$ ' is generated from a bivariate normal distribution with parameters $\left(\mu_{y}, \sigma_{y}, \mu_{x}, \sigma_{x}, \rho\right)$. Using this sample the values of the various estimators of population C.V are computed. The MSE of the various estimators are computed using 10,000 replicated samples. The configurations used in the simulations are as follows:

The values of the C.V of the study variable used in the simulations were $0.1,0.3,0.5,0.8$, 1.0 and 2.0. For each fixed value of C.V of the study variable, a set of four values of C.V of the auxiliary variable are considered. They are 0.5, 1.0, 1.5 and 2.0 times the C.V of the study variable. The correlation co-efficient ' $r$ ' used in the simulation study are -0.9 , $-0.7,-0.5,-0.3,-0.1,0,0.1,0.3,0.5,0.7,0.9$. The sample sizes considered are $n=100,200$.

The total number of configurations works out to be $6 * 4 * 11 * 2=528$.

\subsection{Finite sample MSE}

This paper evaluates the performance of 28 estimators of C.V over 528 configurations. In order to facilitate a meaningful inference, the results are tabulated by taking the average of the mean square error for each of the estimators across the four values of C.V of the auxiliary variable. The results are carefully examined for each value of the C.V of the study variable over various value of the correlation co-efficient between the study and auxiliary variable. To save space, the best eight estimators (having smaller mean square error) are identified. Table (4.1) represent the relative efficiency of the 8 best estimators compared to sample C.V, $\hat{\theta}_{y_{1}}$ when the sample size $n=100$ across all correlation coefficient when C.V of the study variable are $0.1,0.3,0.5$ and 1.0. The following conclusions emerge.

\begin{tabular}{|c|c|c|c|c|c|c|c|c|c|c|c|c|}
\hline \multirow{2}{*}{\multicolumn{2}{|c|}{$\begin{array}{l}\text { C. V of the study } \\
\text { variable }\end{array}$}} & \multicolumn{11}{|c|}{ Correlation co-efficient ' $r$ ' $(n=100)$} \\
\hline & & \multirow{2}{*}{$\begin{array}{c}\mathbf{- 0 . 9} \\
100\end{array}$} & \multirow{2}{*}{$\begin{array}{c}\mathbf{- 0 . 7} \\
100\end{array}$} & \multirow{2}{*}{$\begin{array}{c}\mathbf{- 0 . 5} \\
100\end{array}$} & \multirow{2}{*}{$\begin{array}{c}\mathbf{- 0 . 3} \\
100\end{array}$} & \multirow{2}{*}{$\begin{array}{c}-\mathbf{- 0 . 1} \\
100\end{array}$} & \multirow{2}{*}{$\begin{array}{c}\mathbf{0} \\
100\end{array}$} & \multirow{2}{*}{$\begin{array}{l}\mathbf{0 . 1} \\
100\end{array}$} & \multirow{2}{*}{$\begin{array}{l}\mathbf{0 . 3} \\
100\end{array}$} & \multirow{2}{*}{$\begin{array}{c}\mathbf{0 . 5} \\
100\end{array}$} & \multirow{2}{*}{$\begin{array}{l}\mathbf{0 . 7} \\
100\end{array}$} & \multirow{2}{*}{$\begin{array}{c}\mathbf{0 . 9} \\
100\end{array}$} \\
\hline \multirow{9}{*}{0.1} & $\hat{\theta}_{1}$ & & & & & & & & & & & \\
\hline & $\hat{\theta}_{3}$ & 358.3 & 326.67 & 272.5 & 96.87 & 98.68 & 98.89 & 97.62 & 95.57 & 269.82 & 330.01 & 340 \\
\hline & $\hat{\theta}_{2}$ & 300 & 286.67 & 213.13 & 95.42 & 97.56 & 97.60 & 97.01 & 94.89 & 222.25 & 262.08 & 280.50 \\
\hline & $\hat{\theta}_{16}$ & 275 & 250.11 & 215.67 & 94.34 & 95.67 & 99.78 & 94.58 & 93.89 & 210.08 & 242.50 & 276.67 \\
\hline & $\hat{\theta}_{18}$ & 236.80 & 239.42 & 212.13 & 91.56 & 90.98 & 98.10 & 91.47 & 92.70 & 208.35 & 222.25 & 242.50 \\
\hline & $\hat{\theta}_{9}$ & 200 & 182.30 & 150 & 80 & 75 & 98.0 & 78.89 & 82.28 & 161.13 & 170.01 & 233.33 \\
\hline & $\hat{\theta}_{12}$ & 200 & 171.66 & 150 & 75 & 66.67 & 99.01 & 69.89 & 76.67 & 158.67 & 165.67 & 228.76 \\
\hline & $\hat{\theta}_{4}$ & 133.33 & 125 & 110 & 62.27 & 58.84 & 99.08 & 60 & 69.98 & 107.32 & 129.18 & 142.42 \\
\hline & $\hat{\theta}_{5}$ & 125 & 119.87 & 105.54 & 61.14 & 50 & 98.89 & 52.25 & 62.58 & 108.56 & 119.88 & 129.80 \\
\hline
\end{tabular}




\begin{tabular}{|c|c|c|c|c|c|c|c|c|c|c|c|c|}
\hline \multirow{9}{*}{0.3} & $\hat{\theta}_{1}$ & 100 & 100 & 100 & 100 & 100 & 100 & 100 & 100 & 100 & 100 & 100 \\
\hline & $\hat{\theta}_{3}$ & 372.8 & 333.34 & 280.56 & 97.67 & 98.90 & 99.01 & 97.65 & 94.57 & 289.82 & 340.05 & 382.46 \\
\hline & $\hat{\theta}_{2}$ & 350 & 290.45 & 229.92 & 96.48 & 98.56 & 98.89 & 97.78 & 97.42 & 238.84 & 275.67 & 298.80 \\
\hline & $\hat{\theta}_{16}$ & 292.34 & 268.11 & $223 . .47$ & 93.45 & 96.57 & 98.78 & 95.58 & 94.49 & 220.21 & 253.54 & 283.56 \\
\hline & $\hat{\theta}_{18}$ & 276.67 & 245.64 & 218.33 & 93.48 & 92.89 & 98.10 & 92.87 & 94.45 & 215.32 & 235.36 & 254.60 \\
\hline & $\hat{\theta}_{9}$ & 222.32 & 190.36 & 165.02 & 88.98 & 79.01 & 98.67 & 80.50 & $84 . .27$ & 170.25 & 182.30 & 242.46 \\
\hline & $\hat{\theta}_{12}$ & 217.18 & 182.64 & 160 & 84.49 & 75 & 97.02 & 72.90 & 79.89 & 166.67 & 175 & 232.58 \\
\hline & $\hat{\theta}_{4}$ & 142.23 & 129.65 & 120.09 & 74.42 & 65.57 & 98.07 & 66.89 & 72.18 & 115.87 & 128.32 & 158.89 \\
\hline & $\hat{\theta}_{5}$ & 130.09 & 122.98 & 110 & 68.34 & 61.17 & 97.68 & 63.45 & 69.98 & 112.65 & 120.90 & 135.43 \\
\hline \multirow{9}{*}{0.5} & $\hat{\theta}_{1}$ & 100 & 100 & 100 & 100 & 100 & 100 & 100 & 100 & 100 & 100 & 100 \\
\hline & $\hat{\theta}_{3}$ & 350.28 & 320.45 & 265.58 & 95.87 & 96.80 & 98.38 & 95.67 & 96.82 & 254.65 & 310.18 & 340.09 \\
\hline & $\hat{\theta}_{2}$ & 300 & 276.37 & 224.02 & 94.89 & 93.15 & 97.54 & 93.58 & 92.36 & 222.18 & 269.98 & 281.56 \\
\hline & $\hat{\theta}_{16}$ & 272.28 & 252.18 & 215.67 & 92.64 & 91.68 & 98.87 & 92.67 & 93.45 & 208.89 & 238.64 & 269.87 \\
\hline & $\hat{\theta}_{18}$ & 243.18 & 236.67 & 210.56 & 91.45 & 92.38 & 98.60 & 93.18 & 92.06 & 205.89 & 224.56 & 249.76 \\
\hline & $\hat{\theta}_{9}$ & 210 & 186.87 & 160 & 85 & 78.89 & 97.84 & 88.87 & 83.49 & 153.58 & 176.67 & 230.52 \\
\hline & $\hat{\theta}_{12}$ & 200 & 168.87 & 148.89 & 71.67 & 65.45 & 97.10 & 66.58 & 70.07 & 150.87 & 168.64 & 218.98 \\
\hline & $\hat{\theta}_{4}$ & 128.36 & 119.18 & 112.36 & 66.45 & 59.60 & 96.68 & 61.02 & 67.41 & 113.15 & 120.08 & 135.68 \\
\hline & $\hat{\theta}_{5}$ & 125 & 115.56 & 108.24 & 63.18 & 55.89 & 97.78 & 54.02 & 64.89 & 106.69 & 116.67 & 128.45 \\
\hline \multirow{9}{*}{1.0} & $\hat{\theta}_{1}$ & 100 & 100 & 100 & 100 & 100 & 100 & 100 & 100 & 100 & 100 & 100 \\
\hline & $\hat{\theta}_{3}$ & 340.87 & 310.67 & 268.87 & 93.38 & 94.38 & 98.87 & 93.78 & 92.56 & 250.45 & 298.46 & 336.67 \\
\hline & $\hat{\theta}_{2}$ & 305.48 & 270.26 & 220.08 & 92.16 & 94.89 & 97.41 & 94.52 & 92.08 & 230.08 & 254.76 & 270.02 \\
\hline & $\hat{\theta}_{16}$ & 285.67 & 256.75 & 218.92 & 91.18 & 92.56 & 98.76 & 92.01 & 91.76 & 210.58 & 236.67 & 265.45 \\
\hline & $\hat{\theta}_{18}$ & 246.51 & 239.02 & 212.75 & 92 & 92.79 & 98.60 & 93.18 & 92.18 & 208.75 & 225.36 & 250.08 \\
\hline & $\hat{\theta}_{9}$ & 208.67 & 190.04 & 181.46 & 88.87 & 82.47 & 98.0 & 84.65 & 87.64 & 168.98 & 184.39 & 220.86 \\
\hline & $\hat{\theta}_{12}$ & 203.32 & 170.78 & 150.67 & 74.58 & 70.82 & 97.02 & 72.69 & 78.26 & 155.25 & 169.50 & 215.54 \\
\hline & $\hat{\theta}_{4}$ & 135.34 & 125 & 120.08 & 65.48 & 60.10 & 96.16 & 62.76 & 69.91 & 115.56 & 128.87 & 140.65 \\
\hline & $\hat{\theta}_{5}$ & 129.87 & 119.67 & 110.35 & 61.90 & 54.67 & 97.26 & 56.89 & 65.40 & 108.24 & 120.06 & 131.68 \\
\hline
\end{tabular}

Table (4.1): Relative efficiency of the eight best estimators compared to the sample C.V $\left(\hat{\theta}_{y_{1}}\right)$ 
From the table it is clear that when the correlation co-efficient is moderate to high $(\mathrm{r}= \pm 0.9, \pm 0.7, \pm 0.5)$, the estimator which has got the maximum relative efficiency corresponds to $\left(\hat{\theta}_{y_{3}}\right)$ where information on C.V of the auxiliary variable is used followed by the ratio type regression estimator $\left(\hat{\theta}_{y_{2}}\right)$ where the regression estimators of mean and variance are used to estimate $\theta_{y}$. The other estimators are the estimator proposed by Patel and Shah, i.e. $\left(\hat{\theta}_{y_{16}}\right)$ and the regression estimator constructed from the optimum estimator of Tripathi et al., (2002), namely ( $\hat{\theta}_{y_{18}}$ ) also emerges as the best estimators. Their positions in terms of relative ranking of the estimators are 3 and 4 respectively; ranking one corresponds to the estimator having maximum relative efficiency. Two of the estimators proposed by Tripathi et al., (2002) also emerge as the best estimators. The estimators $\hat{\theta}_{y_{9}}$ and $\hat{\theta}_{y_{12}}$ (in the notations of Tripathi et al., (2002), $e_{6}$ and $e_{7}$ ) emerge as the fifth and sixth best estimators. The other best estimators are the estimators proposed by Archana and Rao (2011); they are the ratio estimator $\hat{\theta}_{y_{4}}$, where information on mean of the auxiliary variable is used and the ratio estimator $\hat{\theta}_{y_{5}}$, where information on C.V of the auxiliary variable is used. The table also indicates that, the relative efficiency of the various estimators' increases as the value of C.V of the study variable starts increasing from 0.1 to 0.3 and remains constant thereafter.

We have also examined the relative efficiency of the various estimators of C.V across the correlation co-efficient for each value of C.V of the study variable. When the correlation is moderate to high $(\mathrm{r}= \pm 0.9, \pm 0.7, \pm 0.5)$, the relative efficiency of the estimators are high and decreases for moderate or low values of the correlation co-efficient. As the relative efficiency is the ratio of two mean square error's, they do not exactly indicate how the performance of an estimator is affected by the correlation co-efficient. For this purpose it is necessary to examine the mean square error of the various estimators. To save space the table of mean square error's are not reported here. The examination of the mean square error for the various estimators indicates that the mean square error increases when the correlation co-efficient ranges from -0.9 to 0 and starts declining as the correlation co-efficient increases to 0.9 (except for sample C.V which does not depend on the correlation co-efficient). Careful examination of the mean square error also reveals that the best estimators perform well when the study and auxiliary variable are highly correlated and when there is low correlation sample C.V emerges as the best estimator.

The examination of the table also indicates that, the relative efficiency of the various regression and ratio estimators of $\mathrm{C} . \mathrm{V}$ decreases when the correlation co-efficient is low compared to the sample C.V. This conclusion is in line with the performance of these estimators in finite population. An important conclusion that needs to be highlighted is that the decrease in the relative efficiency is marginal compared to the sample C.V. For eg: For the best estimator $\hat{\theta}_{y_{3}}$, the relative efficiency ranges between $(95.57,98.68)$, when the correlation co-efficient ranges between -0.3 to 0.3 and C.V of the study variable is equal to 0.1 . For the second best estimator $\hat{\theta}_{y_{2}}$, the corresponding relative efficiency ranges from 94.89 to 97.56 . Even when the correlation co-efficient is 0 , the relative 
efficiency of these 2 estimators compared to sample C.V are 97.60 and 98.89 respectively. Thus the previous results indicate that these 2 estimators can safely be used when no knowledge is available regarding the correlation co-efficient. Among the other best estimators, this conclusion remains the same for the estimator of Patel and Shah $\left(\hat{\theta}_{y_{16}}\right)$ and the regression estimator constructed from the optimum estimator of Tripathi et al., (2002), namely $\left(\hat{\theta}_{y_{18}}\right)$.

In this investigation an attempt is also made to study the impact of C.V of the auxiliary variable on the efficiency of the various estimators. For fixed value of C.V of the study variable and the correlation co-efficient, the mean square error is minimum for any estimator when the C.V of the auxiliary variable is lower or equal to the C.V of the study variable compared to higher values of $\mathrm{C}$.V of the auxiliary variable. To save space the table is not reported here.

\subsection{Discussions}

In this paper we have proposed various estimators of C.V in finite population (when the sampling design is SRSWR) as estimators of CV for a bivariate normal distribution. In addition to the estimator available in the literature, we have constructed 12 new estimators of C.V using the optimum estimators of Tripathi et al., (2002). Among these 12 estimators only one estimator namely $\hat{\theta}_{y_{18}}$ emerge as one of the best estimator. The optimum estimator of Tripathi et al.,(2002) does not emerge as one of the best eight estimators. The reason is that here we are estimating the finite sample mean square error which is an accurate estimate of the mean square error and the optimality criterion is asymptotic in nature. On the other hand, two other estimators of Tripathi et al., (2002) (i.e $\hat{\theta}_{y_{9}}$ and $\hat{\theta}_{y_{12}}$ ), emerges as the best estimators. The other salient finding is that the simple regression estimator using C.V of the study variable emerges as the best estimator. This result emphasizes the fact that additional information on the population mean or variance of the auxiliary variable does not improve the performance of the estimators. This conclusion is with special reference to the estimators $\left(\hat{\theta}_{y_{13}}\right.$ and $\left.\hat{\theta}_{y_{14}}\right)$ of Tripathi et al., (2002).

Archana and Rao (2011) observed that one can always improve any estimator of a parameter by constructing a regression estimator using this estimator. In this paper we have constructed 12 new estimators of C.V using the optimum estimator of Tripathi et al (2002). Among these 12 estimators, only one estimator namely $\hat{\theta}_{y_{18}}$ emerges as one of the best estimators and ranks in fourth position in terms of relative efficiency. This indicates that improved estimators have to be constructed with caution if at all.

\section{Conclusion}

In this paper, we propose 28 estimators of $\mathrm{C} . \mathrm{V}$ of a component of bivariate normal distributions when additional information is available regarding the parameter of the second component. The sample C.V of the component under consideration is the 
maximum likelihood estimator of the C.V of the component from a bivariate normal distribution. When the correlation co-efficient is moderate to high, 8 of the proposed estimators have a smaller mean square error compared to the maximum likelihood estimator of C.V. Even when the correlation co-efficient is low, the performance in terms of relative efficiency is marginally lower compared to the maximum likelihood estimator. The best two estimators are the regression estimator using information on C.V of the auxiliary variable and regression type estimator where improved estimator of mean \&

variance are used to estimate the ratio $\theta_{y}=\frac{\sigma_{y}}{\mu_{y}}$. Since the latter estimator needs addition information regarding mean and variance of the auxiliary variable, while the former needs information only on the $\mathrm{CV}$ of the auxiliary variable. Thus we recommend this estimator $\left(\hat{\theta}_{y_{3}}\right)$ when paired observations are available from a bivariate normal distribution. The finite sample estimate of the mean square error indicates that the maximum likelihood estimators as well as the other best 8 estimators are admissible. Further the numerical results indicate that, no estimator attains the CR type lower bound. This is in contrast to the conclusion that the asymptotic mean square error of sample C.V (the maximum likelihood estimator) attains the C.R lower bound for a univariate normal distribution.

\section{Acknowledgements}

This paper is part of the researcher's Ph.D work under the guidance of Dr. K. Aruna Rao. Professor Rao is legally blind and I express my deep gratitude to him for suggesting the problem and for his valuable guidance.

\section{References}

1. Ahmed, S.E. (2002). Simultaneous estimation of Co-efficient of Variation, Journal of Statistical Planning and Inference, 104, 31-51.

2. Ananthakrishnan, R. and Soman, M.K. (1989). Statistical distribution of daily rainfall and its association with the co-efficient of variation of rainfall series, International Journal of Climatology, 9, 485-500.

3. Bennett, B.M. (1976). On an approximate test for homogeneity of co-efficients of variation. In: Walter John Zieglir, Birhauser Verlag, Basel, Stuttgart, eds., Contribution to Applied Statistics, 169-171.

4. Bhat, K. and Rao, K.A. (2011). Inference for Normal mean with known Coefficient of Variation, Published Ph.D thesis by LAP LAMBERT Academic Publishing, Germany.

5. Breunig, R. (2001). An almost unbiased estimator of the co-efficient of variation. Economics Letters, 70(1), 15-19.

6. Brief, R.P. and Owen, J. (1969). A Note on earnings risk and the co-efficient of variation, J. Finance, 24, 901-904.

7. Chaturvedi, A. and Rani, U. (1996). Fixed width confidence interval estimation of Inverse co-efficient of variation in normal population. Microelectronics and Reliabiliy, 36, 1305-1308. 
8. Curto, J.D. and Pinto, J.C. (2009). The co-efficient of variation asymptotic distribution in the case of non-iid random variables, Journal of Applied Statistics, 36(1), 21-32.

9. Das, A.K. and Tripathi, T.P. (1981 a). Sampling strategies for coefficient of variation using knowledge of the mean using an auxiliary character. Tech. Rep. Stat. Math. 5/81. ISI, Calcutta.

10. Das, A.K. and Tripathi, T.P. (1981 b). A class of estimators for co-efficient of variation using knowledge on coefficient of variation of an auxiliary character. Paper presented at annual conference of Ind. Soc. Agricultural Statistics. Held at New Delhi, India.

11. Das, A.K. and Tripathi, T.P. (1992-93). Use of auxiliary information in estimating the co-efficient of variation. Alig. J. of. Statist., 12 and 13, 51-58.

12. Doornabos, R and Dijkstra, J.B. (1983). A multi sample test for the equality of coefficient of variation in normal population. Common. Statist-Simula. Computa, 12, 147-158.

13. Fieller, E.C. (1932). A numerical test of the adequacy of A. T. McKay's approximation. J. Roy. Stat. Soc. 95, 699-702.

14. Forkman, J. (2009). Estimator and tests for common co-efficients of variation in normal distributions. Communications in statistics-Theory and Methods, 38(2), 233-251.

15. Gupta, C.R and Ma, S. (1996). Testing the equality of Co-efficient of Variation in 'k' Normal populations. Common Statist. Theory Meth, 25, 115-132.

16. Hendricks, W.A. and Robey, W.K. (1936). The sampling distribution of the Coefficient of variation. Annals. Math. Stat. (7), 129-132.

17. Lehmann, E.L. and Casella, G. (1998). Theory of point estimation. Second edition, Springer-Verlag, New York, Inc.

18. Liu, W., Pang, W.K. and Huang, W.K. (2006). Exact confidence bounds for the co-efficient of variation of a normally distributed population, International. J. of Statistics and Systems, 1(1), 81-86.

19. Mahmoudvand, R. and Hassani, H. (2007). Two new confidence intervals for the C.V in a normal distribution. Journal of Applied Statistics, 36: 4, 429-442.

20. Maiti, P. (2009). Some shrinkage-type estimators for variances of univariate populations. J. Ind. Soc. Prob. Statistics, 11, 55-77.

21. Maiti, P. and Tripathi, T.P. (2009). Some improved variance estimators from a bivariate non-normal population. Pak. J. Statist, 25(2), 165-194.

22. McKay, A.T. (1932). Distribution of the Co-efficient of variation and the extended 't' distribution. J.Royal.Stat.Soc., 95, 696-698.

23. Miller, E.G. and Karson, M.J. (1977). Testing equality of two co-efficients of variation, Am. Stat. Assoc. Proc. Bus. Econ. Sect. Part I, 278-283.

24. Nairy, K.S. and Rao, K.A. (2003). Tests of coefficient of variation in normal population. Communications in Statistics: Simulations and Computation, 32 (3), $641-661$.

25. Ng, C.K. (2006). Performance of three methods of interval estimation of the coefficient of variation, InterStat. 
26. Patel, P. A. and Shah Rina. (2009). A Monte Carlo comparison of some suggested estimators of Co-efficient of variation in finite population. Journal of Statistics sciences, 1(2), 137-147.

27. Pearson, E.S. (1932). Comparison of A.T. McKay's approximation with experimental sampling results. J.Royal.Stat.Soc. 95, 703-704.

28. Panichkitkosolkul, W. (2009). Improved confidence intervals for a Co-efficient of variation of a normal distribution, Thailand statistician, 7(2), 193-199.

29. Rajyaguru, A. and Gupta, P. (2006). On the estimation of the co-efficient of variation from finite population-II, Model Assisted Statistics and application, 1(1), 57-66.

30. Rajyaguru, A. and Gupta, P. (2002). On the estimation of the co-efficient of variation from finite population-I, Model Assisted Statistics and application, 36(2), 145-156.

31. Rao, A.K. and Bhatta, A.R.S. (1989). A note on test for Co-efficient of Variation. Calcutta Statistical Association Bulletin, 38, 151-152.

32. Rao, A.K. and Vidya, R. (1992). On the performance of a test for co-efficient of variation. Calcutta Statistical Association Bulletin, 42, 87-95.

33. Shafer, N.J and Sullivan, J.A. (1986). A simulation study of a test for the Equality of Co-efficient of variation. Common. Statist-Simula. Computa, 15, 681-695.

34. Singh, M. (1993). Behavior of sample Co-efficient of Variation drawn from several distributions. Sankhya. B. 55, 65-76.

35. Tripathi, T.P. Singh, H.P. and Upadhyaya, L.N. (2002). A general method of estimation and its application to the estimation of co-efficient of variation. Statistics in Transition, 5(6), 887-909.

36. Verril, S.P. and Johnson, R.A. (2007). Confidence bounds and hypothesis tests for normal distribution, Research Paper FPL-RP-638, Madison, WI: U.S. Department of Agriculture, Forest Service, Forest Products Laboratory. 57.

37. Weinraub, H.J. and Kuhlman, B.R. (1994). The effect of common stock beta variability on the variability of portfolio beta, J. Finance. Strateg. Decis. 7(2), 79-84. 


\section{Appendix A}

$$
\begin{aligned}
& M\left(\hat{\theta}_{y_{1}}\right)=\left\{\frac{3 \sigma_{y y}}{4 n \mu_{y}^{2}}-\frac{(n-3) \sigma_{y y}}{4 n(n-1) \mu_{y}^{2}}+\frac{1}{n} \theta_{y}^{4}\right\}+O\left(\frac{1}{n^{2}}\right) \\
& M\left(\hat{\theta}_{y_{2}}\right)=\left\{\begin{array}{l}
\frac{1}{n} \theta_{y}^{4}+\frac{B_{1}^{2}}{n} \frac{\sigma_{x x}}{\mu_{y}^{2}} \theta_{y}^{2}+\frac{3 \sigma_{y y}}{4 n \mu_{y}^{2}}-\frac{(n-3)}{4 n(n-1)} \theta_{y}^{2}+\frac{3 B_{2}^{2}\left(\sigma_{x x}\right)^{2}}{4 n \sigma_{y y} \mu_{y}^{2}}-\frac{(n-3) B_{2}^{2}\left(\sigma_{x x}\right)^{2}}{4 n(n-1) \sigma_{y y} \mu_{y}^{2}} \\
-2 B_{1} \theta_{y}^{2} \frac{\sigma_{x y}}{\mu_{y}^{2}}-\frac{B_{2} \sigma_{x x}}{2 n \mu_{y}^{2}}-\frac{B_{2}\left(\sigma_{x y}\right)^{2}}{n \sigma_{y y} \mu_{y}^{2}}+\frac{B_{2} \sigma_{x x}}{2 n \mu_{y}^{2}}
\end{array}\right\}+O\left(\frac{1}{n^{2}}\right) \\
& M\left(\hat{\theta}_{y_{3}}\right)=\left\{\begin{array}{l}
\frac{1}{n} \theta_{y}^{4}+\frac{B_{5}^{2}}{n} \theta_{x}^{4}+\frac{3 \sigma_{y y}}{4 n \mu_{y}^{2}}-\frac{(n-3)}{4 n(n-1)} \theta_{y}^{2}+\frac{3 B_{5}^{2} \sigma_{x x}}{4 n \mu_{x}^{2}}-\frac{(n-3) B_{5}^{2} \sigma_{x x}}{4 n(n-1) \mu_{x}^{2}}-\frac{2 B_{5} \sigma_{x y} \sigma_{x} \sigma_{y}}{n \mu_{x}^{2} \mu_{y}^{2}} \\
-\frac{B_{5} \sigma_{x x} \sigma_{y y}}{2 n \sigma_{x} \sigma_{y} \mu_{x} \mu_{y}}-\frac{B_{5}\left(\sigma_{x y}\right)^{2}}{n \sigma_{x} \sigma_{y} \mu_{x} \mu_{y}}-\frac{B_{5} \sigma_{x x} \sigma_{y y}}{2 n \sigma_{x} \sigma_{y} \mu_{x} \mu_{y}}
\end{array}\right\}+O\left(\frac{1}{n^{2}}\right) \\
& M\left(\hat{\theta}_{y_{4}}\right)=\left\{\frac{1}{n} \theta_{y}^{4}+\frac{1}{n} \theta_{x}^{2} \theta_{y}^{2}+\frac{3}{4 n} \theta_{y}^{2}-\frac{(n-3)}{4 n(n-1)} \theta_{y}^{2}+\frac{2 \sigma_{x y}}{n \mu_{x} \mu_{y}} \theta_{y}^{2}\right\}+O\left(\frac{1}{n^{2}}\right) \\
& M\left(\hat{\theta}_{y_{5}}\right)=\left\{\frac{1}{n} \theta_{y}^{4}+\frac{3}{2 n} \theta_{y}^{2}-\frac{(n-3)}{2 n(n-1)} \theta_{y}^{2}+\frac{1}{n} \theta_{x}^{2} \theta_{y}^{2}-\frac{2 \sigma_{x y}}{n \mu_{x} \mu_{y}} \theta_{y}^{2}-\frac{\left(\sigma_{x y}\right)^{2}}{n \mu_{y}^{2} \sigma_{x x}}\right\}+O\left(\frac{1}{n^{2}}\right) \\
& M\left(\hat{\theta}_{y_{9}}\right)=\left\{\frac{3}{4 n} \theta_{y}^{2}-\frac{(n-3)}{4 n(n-1)} \theta_{y}^{2}+\frac{3}{4 n} \frac{\alpha_{2}^{2} \sigma_{x x}}{\bar{Y}^{2}}-\frac{(n-3)}{4 n(n-1)} \frac{\alpha_{2}^{2} \sigma_{x x}}{\bar{Y}^{2}}+\frac{1}{n} \theta_{y}^{4}-\frac{\alpha_{2} \sigma_{x y}^{2}}{n \sigma_{x} \sigma_{y} \bar{Y}^{2}}\right\}+O\left(\frac{1}{n^{2}}\right) \\
& M\left(\hat{\theta}_{y_{12}}\right)=\left\{\frac{3}{4 n} \theta_{y}^{2}-\frac{(n-3)}{4 n(n-1)} \theta_{y}^{2}+\frac{3}{4 n} \frac{\sigma_{x x}}{\bar{Y}^{2}}-\frac{(n-3)}{4 n(n-1)} \frac{\sigma_{x x}}{\bar{Y}^{2}}+\frac{1}{n} \theta_{y}^{4}-\frac{\sigma_{x y}{ }^{2}}{n \sigma_{x} \sigma_{y} \bar{Y}^{2}}\right\}+O\left(\frac{1}{n^{2}}\right) \\
& M\left(\hat{\theta}_{y_{18}}\right)=\left\{\frac{3}{4 n} \theta_{y}^{2}-\frac{(n-3)}{4 n(n-1)} \theta_{y}^{2}+\frac{3}{n}\left(\sigma_{x x}\right)^{2}\left(\hat{\delta}_{2}+B_{7}\right)^{2}-\frac{(n-3)}{n(n-1)}\left(\sigma_{x x}\right)^{2}\left(\hat{\delta}_{2}+B_{7}\right)^{2}+\frac{1}{n} \sigma_{x x} \hat{\delta}_{1}^{2}\right. \\
& \left.+\frac{1}{n} \theta_{y}^{4}-\frac{2 \sigma_{x y}{ }^{2}\left(\hat{\delta}_{2}+B_{7}\right)}{n \sigma_{y} \bar{Y}}+\frac{2 \sigma_{x y} \sigma_{y} \hat{\delta}_{1}}{n \bar{Y}^{2}}\right\}+O\left(\frac{1}{n^{2}}\right)
\end{aligned}
$$

